hep-th/9910172

IASSNS-HEP-99/97

RUNHETC-99-39

\title{
D-Branes and Bundles on Elliptic Fibrations
}

\author{
Duiliu-Emanuel Diaconescu ${ }^{\natural}$ and Christian Römelsberger ${ }^{\sharp}$ \\ घ School of Natural Sciences \\ Institute for Advanced Study \\ Olden Lane, Princeton, NJ 08540 \\ diacones@sns . ias.edu \\ $\sharp$ Department of Physics and Astronomy \\ Rutgers University \\ Piscatway, NJ 08555 \\ roemel@physics . rutgers . edu
}

We study the D-brane spectrum on a two-parameter Calabi-Yau model. The analysis is based on different tools in distinct regions of the moduli space: wrapped brane configurations on elliptic fibrations near the large radius limit, and SCFT boundary states at the Gepner point. We develop an explicit correspondence between these two classes of objects, suggesting that boundary states are natural quantum generalizations of bundles. We also find interesting D-brane dynamics in deep stringy regimes. The most striking example is, perhaps, that nonsupersymmetric D6-D0 and D4-D2 large radius configurations become stable BPS states at the Gepner point.

October 1999 


\section{Introduction}

Since their discovery [1], D-branes have been an ubiquitous presence in string dualities and $\mathrm{M}$ theory. However, in spite of a detailed understanding of D-brane dynamics in flat space, their behavior in abstract conformal field theories is less understood. A particular class of string vacua where D-brane spectra are especially interesting consists of $(2,2)$ superconfomal field theories (SCFT). The moduli spaces of $(2,2)$ SCFT's are usually affected by stringy quantum corrections which result in a very rich phase structure. One typically has geometric phases, where classical geometry can be used at least as a guiding principle in describing the physics, and nongeometric phases where the semiclassical description breaks down. It is known that the closed string quantum corrections result in a quantum deformation of the classical cohomology rings of varieties. Loosely, one can think of chiral primary operators in abstract SCFT's as representing quantum deformations of classical cohomology cycles.

Adding D-branes is essentially equivalent to adding boundaries (with appropriate boundary conditions) to the string worldsheet. The coupling to the open string sector adds new ingredients to the space-time physics. In geometric phases, the new degrees of freedom can be described semiclassically as gauge fields living on various submanifolds of space-time. Therefore, as explained in [2,3], in a geometric phase, D-branes are naturally described as K theory classes rather than singular cohomology classes. However, such an explicit and intuitive description is lacking in deep stringy regimes. Given the breakdown of the classical geometry in these regions, one has to rely on abstract SCFT techniques (whenever possible) in order to classify D-brane charges and study their dynamics. An effective approach to this problem relies on the formalism of boundary states, which can be loosely thought as closed string coherent states solving the SCFT boundary conditions. In this context, boundary states seem to be the natural quantum deformations of vector bundles.

The main point of the present paper is the interplay between the two different descriptions of D-branes in $N=2$ string vacua. The natural framework, which insures an explicit description in both regimes, consists of Calabi-Yau compactifications continuously connected to Gepner models. Since the latter are exactly solvable SCFT's one can construct explicit boundary states solving Cardy's consistency condition [4, [6, 6]. This offers detailed information on part of the spectrum of branes in a nongeometric phase. As outlined above, we would like to understand if these states have a definite geomet- 
ric interpretation in terms of D-branes wrapping supersymmetric cycles in a Calabi-Yau manifold of very large radius. Restricting our attention to the charges of BPS states (i.e. ignoring dynamical aspects such as stability and existence of bound states) this question can be systematically answered once the exact special geometry of the moduli space is known. The map between the symplectic charge lattices can be found using the boundary state representation of the symplectic intersection form described in [7]. Note that this map allows one to determine the effective BPS charges of the Gepner model boundary states as seen by a low energy observer. For B type boundary states [8] (which correspond to D-branes wrapping even homology cycles), these are not the same as the microscopic D-brane charges, since the former include gravitational corrections [9, 10, 11, 2, 12]. In such cases, the relation between the microscopic and effective charges involves the computation of a Mukai vector. The question of relating physical BPS states in the two regimes is more subtle and it requires a more detailed knowledge of the geometry of the Calabi-Yau variety near the large radius limit, including detailed results on the classification of vector bundles and special lagrangian cycles.

In order to obtain concrete results, we focus on B type boundary states in the two parameter model $\mathbf{P}_{4}^{1,1,1,6,9}[18]$ of [13]. After resolving the singularities, these hypersurfaces exhibit a structure of elliptically fibered Calabi-Yau varieties which facilitates the construction of the relevant holomorphic bundles (or sheaves). Exploiting this feature, we explicitly analyze moduli and stability questions of Gepner model BPS states in the large radius limit. Similar issues have been addressed in [14] for the quintic and [15] for local orbifold models.

At the Gepner point, the boundary states are generically organized in orbits of a discrete symmetry group (in the present model $\mathbf{Z}_{18}$, as detailed in section four). We find that, within a given orbit, certain Gepner model states become unstable in the geometric region, signaling the crossover of a wall of marginal stability. At the same time, an important fraction of states in the same orbit are supersymmetric and stable in the large radius limit. This shows that the discrete $\mathbf{Z}_{18}$ symmetry is not a good symmetry in the geometric phase.

Perhaps the most striking examples consist of the nonsupersymmetric D6 + D0 and respectively D4 + D2 states, which are repulsive in the large volume limit. On the other hand, they can be shown to correspond to supersymmetric Gepner model boundary states, giving explicit examples for the transitions predicted in [14]. This result is particularly 
interesting when interpreted in terms of D3-branes wrapping middle homology cycles in the mirror manifold. According to [16], mirror symmetry can be thought as T-duality on the $T^{3}$ fibers of a special lagrangian fibration. A Calabi-Yau hypersurface $X$ near the large

radius limit is mapped to a mirror manifold $\hat{X}$ in a neighborhood of the large complex structure limit. This transformation maps the D0-brane to a D3-brane wrapping the $\hat{T}^{3}$ fibers of the dual fibration, while the D6-brane is mapped to a D3-brane wrapping the base $B$ of the fibration. Therefore our analysis shows that for $\hat{X}$ in a certain neighborhood of the large complex structure limit, there should not exist any special lagrangian cycle in the homology class $\hat{T}^{3}+B$. However, in a different region of the complex structure moduli space of $\hat{X}$, that maps to a neighborhood of the Gepner point, the same homology class should actually contain a special lagrangian cycle that corresponds to the supersymmetric Gepner model boundary state. This provides a concrete example, in a compact Calabi-Yau space, for the transitions discussed in [17] and from a physical point of view in [18]. It would be very interesting to understand the mathematical details of this transition in the present context, but we leave this for future work.

For the boundary states that correspond to supersymmetric brane configurations, we find a remarkable agreement between the number of moduli computed in the two regions. This suggests that the superpotential couplings considered in [14] vanish in this model, but we do not check this explicitly. Also, an interesting point is the presence of a Gepner model boundary state with the charge of a single D0-brane (unlike the quintic studied in [14]). This shows that D0-branes are not necessarily a sign of geometrical compactification.

The paper is structured as follows. Section two consists of a brief review of classical and quantum aspects of the two parameter model of [13]. In section three, we explain the relation between microscopic D-branes and BPS states on the moduli space. Section four is devoted to boundary states in the Gepner model, emphasizing the SCFT intersection form and marginal deformations. In section five we tie together all loose ends and construct an explicit map between Gepner boundary states and D-branes.

\section{The Geometry of the Elliptic Model}

This section consists of a brief review of the classical and quantum geometry of the two parameter model of [13]. We focus on facts of direct relevance to the spectrum of BPS states in a neighborhood of the Gepner point and respectively the large radius limit. 


\subsection{Classical Geometry}

The elliptic model $\mathbf{P}_{4}^{(1,1,1,6,9)}[18]$ describes degree 18 hypersurfaces in the weighted projective space $\mathbf{P}_{4}^{(1,1,1,6,9)}$. A simple example of such a hypersurface $X$ is given by the equation

$$
z_{1}^{18}+z_{2}^{18}+z_{3}^{18}+z_{4}^{3}+z_{5}^{2}=0
$$

in the homogeneous coordinates

$$
\left(z_{1}, z_{2}, z_{3}, z_{4}, z_{5}\right)=\left(\lambda z_{1}, \lambda z_{2}, \lambda z_{3}, \lambda^{6} z_{4}, \lambda^{9} z_{5}\right)
$$

¿From this representation, it is easy to see that $\mathbf{P}_{4}^{(1,1,1,6,9)}$ has a singular line along $z_{1}=$ $z_{2}=z_{3}=0$, which intersects $X$ in a single point. Blowing up this singular line gives an exceptional divisor $E$ in $X$. Another divisor $S$ is defined by the first order polynomials in $x_{1}, x_{2}$ and $x_{3}$. These two divisors generate $H_{4}(X, \mathbf{Z})$. The elliptic fibration structure is induced by the linear system $|S|$ generated by $z_{1}, z_{2}$ and $z_{3}$ which maps $X$ to $\mathbf{P}^{2}$. This fibration has a section given by the exceptional divisor $E$. The generic fiber can be proved to be an elliptic curve, whose homology class in $H_{2}(X)$ will be denoted by $h$. The second generator $l$ of $H_{2}(X, \mathbf{Z})$ is the hyperplane class of $E$. For further use, we record the intersection relations

$$
\begin{array}{ll}
h=S^{2}, & l=E \cdot S, \\
h \cdot E=1, & h \cdot S=0, \quad l \cdot E=-3, \quad l, S=1 \\
E^{2}=-9, & E^{2} \cdot S=-3, \quad E \cdot S^{2}=1, \quad S^{3}=0 .
\end{array}
$$

We choose the generatorst of the Kähler cone to be $(E, S)$, so that a generic Kähler class is written $J=t_{1} E+t_{2} S$, where $\left(t_{1}, t_{2}\right)$ are classical coordinates on the Kähler moduli space.

\subsection{Quantum Geometry}

Since the classical structure of the Kähler moduli space of $X$ is deformed by stringy $\alpha^{\prime}$ corrections, exact results can be obtained by considering the complex structure moduli space of the mirror $\hat{X}$. As explained in [13, the mirror family can be obtained by applying

1 Note that this choice is different from that of [13] where the generators are $H=E+3 S$ and $S$. Therefore, their coordinates are related by a linear transformation to ours. The present choice is motivated by the relation to D-brane states which will be explained latter. 
the Greene-Plesser construction [19]. This results in a two parameter family of complex varieties of the form

$$
z_{1}^{18}+z_{2}^{18}+z_{3}^{18}+z_{4}^{3}+z_{5}^{2}-18 \psi z_{1} z_{2} z_{3} z_{4} z_{5}-3 \phi x_{1}^{6} x_{2}^{6} x_{3}^{6}=0
$$

The complex parameters $(\phi, \psi)$ are subject to certain discrete identifications, therefore they are actually coordinates on a cover of the moduli space.

The exact special geometry of this model is described by a six-vector of periods $\Pi \equiv$ $\left(\Pi_{1} \ldots \Pi_{5}\right)^{t}=\left(\mathcal{F}^{0}, \mathcal{F}^{1}, \mathcal{F}^{2}, 1, t_{1}, t_{2}\right)^{t}$ where $\mathcal{F}$ is the $N=2$ prepotential. Using complex inhomogeneous coordinates $\left(t_{1}, t_{2}\right)$ in a neighborhood of the large radius limit, we have the following asymptotic expansion [13]

$$
\left[\begin{array}{l}
\Pi_{1} \\
\Pi_{2} \\
\Pi_{3} \\
\Pi_{4} \\
\Pi_{5} \\
\Pi_{6}
\end{array}\right]=\left[\begin{array}{c}
\mathcal{F}^{0} \\
\mathcal{F}^{1} \\
\mathcal{F}^{2} \\
1 \\
t_{1} \\
t_{2}
\end{array}\right] \sim\left[\begin{array}{c}
\frac{1}{2}\left(3 t_{1}^{3}+3 t_{1}^{2} t_{2}+t_{1} t_{2}^{2}\right)+\frac{17}{4} t_{1}+\frac{3}{2} t_{2} \\
-\frac{1}{2} t_{2}^{2}+\frac{3}{2} t_{2}-\frac{1}{4} \\
-\frac{1}{2}\left(3 t_{1}^{2}+2 t_{1} t_{2}\right)+\frac{3}{2} t_{1}+\frac{3}{2} \\
1 \\
t_{1} \\
t_{2}
\end{array}\right]
$$

Note that $\Pi$ represents the vector of periods of the holomorphic three-form $\hat{\Omega}$ on the mirror manifold $\hat{X}$ with respect to an integral basis of three-cycles. Due to the different choice of generators of the Kähler cone, this basis is not canonical symplectic as in [13]. In particular, the intersection form is given by

$$
I_{L}=\left(\begin{array}{cccccc}
0 & 0 & 0 & -1 & 0 & 0 \\
0 & 0 & 0 & 0 & -1 & -3 \\
0 & 0 & 0 & 0 & 0 & -1 \\
1 & 0 & 0 & 0 & 0 & 0 \\
0 & 1 & 0 & 0 & 0 & 0 \\
0 & 3 & 1 & 0 & 0 & 0
\end{array}\right)
$$

In order to interpolate between Gepner model boundary states and large radius limit branes, the periods (2.5) must be analytically continued to a neighborhood of the Gepner point. The natural basis of periods in this region is described by an overcomplete eighteenvector $\left(\omega_{0}, \ldots, \omega_{17}\right)^{t}$ whose entries are cyclically permuted by the quantum $\mathbf{Z}_{18}$ discrete symmetry of the Gepner model. These periods satisfy the relations

$$
\begin{aligned}
& \omega_{i}+\omega_{i+9}=0, \\
& \omega_{i}-\omega_{i+3}+\omega_{i+6}=0,
\end{aligned}
$$


which leave only six independent periods $\left(\omega_{0}, \cdots, \omega_{5}\right)^{t}$. The periods $\Pi$ and $\omega$ are related by analytic continuation, resulting in $\Pi=m \omega$ with the connection matrix

$$
m=\left(\begin{array}{cccccc}
-1 & 1 & 0 & 0 & 0 & 0 \\
1 & 0 & 0 & -1 & 1 & 0 \\
0 & 1 & 1 & 1 & 0 & 0 \\
1 & 0 & 0 & 0 & 0 & 0 \\
-1 & 0 & 0 & 1 & 0 & 0 \\
2 & 0 & 0 & -2 & 1 & 1
\end{array}\right)
$$

With respect to the basis of periods $\left(\omega_{0}, \ldots, \omega_{5}\right)^{t}$, the intersection form on $H^{3}(\hat{X}, \mathbf{Z})$ takes the form

$$
I_{G}=m^{-1} I_{L} m^{-1 t}=\left(\begin{array}{cccccc}
0 & 1 & -1 & 0 & 0 & 0 \\
-1 & 0 & 1 & -1 & 0 & 0 \\
1 & -1 & 0 & 1 & -1 & 0 \\
0 & 1 & -1 & 0 & 1 & -1 \\
0 & 0 & 1 & -1 & 0 & 1 \\
0 & 0 & 0 & 1 & -1 & 0
\end{array}\right)
$$

Using the relations (2.7), the matrix $I_{G}$ can be expressed in terms of a $\mathbf{Z}_{18}$ shift matrix

$$
I_{G}=-g^{17}\left(1-g^{17}\right)\left(1-g^{12}\right)\left(1-g^{9}\right)
$$

This particular form of the intersection matrix will play an important role in the interpolation between the Gepner point and the large radius limit.

Finally, let us note that the large radius point sits at the intersection of two divisors $G_{1}, D_{\infty}$ on the boundary of the moduli space. [13]. The monodromy matrices about the two divisors, expressed with respect to the basis of periods (2.5), read

$$
S_{1}=\left(\begin{array}{cccccc}
1 & -1 & -3 & 10 & 9 & 3 \\
0 & 1 & 0 & 0 & 0 & 0 \\
0 & 0 & 1 & 0 & -3 & -1 \\
0 & 0 & 0 & 1 & 0 & 0 \\
0 & 0 & 0 & 1 & 1 & 0 \\
0 & 0 & 0 & 0 & 0 & 1
\end{array}\right) \quad S_{2}=\left(\begin{array}{cccccc}
1 & 0 & -1 & 3 & 2 & 0 \\
0 & 1 & 0 & 1 & 0 & -1 \\
0 & 0 & 1 & 0 & -1 & 0 \\
0 & 0 & 0 & 1 & 0 & 0 \\
0 & 0 & 0 & 0 & 1 & 0 \\
0 & 0 & 0 & 1 & 0 & 1
\end{array}\right)
$$

These monodromy transformations correspond to shifting the B-field by the integral cohomology classes $H=E+3 S$ and respectively $S$. In the next section they will be reinterpreted as natural automorphisms of the $\mathrm{K}$ theory group of $X$. This is a simple particular case of a more ambitious program which proposes an identification between the derived category of complexes of sheaves of $X$ and the Fukaya-Floer category of special lagrangian submanifolds of $\hat{X}$. [20,21,22,23]. A systematic and general approach will appear in [24]. 


\section{D-branes and Periods}

In this section we explore the relation between the spectrum of BPS states of this model and microscopic D-brane states in the large volume limit. Before discussing the technical aspects of the problem, a couple of guiding remarks are in order. The BPS charge lattice of the low energy effective theory is an integral symplectic lattice which can be identified with the middle cohomology lattice of the mirror manifold $H^{3}(\hat{X}, \mathbf{Z})$. The central charge corresponding to an integral vector $\left(n_{6}, n_{4}^{1}, n_{4}^{2}, n_{0}, n_{2}^{1}, n_{2}^{2}\right)$ is

$$
Z=n_{6} \Pi_{1}+n_{4}^{1} \Pi_{2}+n_{4}^{2} \Pi_{3}+n_{0} \Pi_{4}+n_{2}^{1} \Pi_{5}+n_{2}^{2} \Pi_{6} .
$$

On the other hand, in the large radius limit, the lattice of microscopic D-brane charges is an integral quadratic lattice identified with the $\mathrm{K}$ theory lattice $K(X)$. The map between these lattices is a nontrivial question in mirror symmetry, being related to the current efforts of extending the mirror principle to Calabi-Yau spaces with bundles. In the present case, we will construct a map between the low energy charges $\mathbf{n}$ and the topological invariants of the $\mathrm{K}$ theory class $\eta$ by exploiting the exact form of D-brane Chern-Simons couplings worked out in [9, 10, 11, 2, 12]. The topological invariants of $\eta$ are simply given by the Chern character $\operatorname{ch}(\eta)$. The effective charges of a D-brane state represented by $\eta$ are measured by the Mukai vector $Q \in H^{0}(X) \oplus H^{2}(X) \oplus H^{4}(X) \oplus H^{6}(X)$ given by

$$
Q=\operatorname{ch}(\eta) \sqrt{\operatorname{Td}(X)}
$$

The central charge associated to this state is then国

$$
Z(t)=\frac{t^{3}}{6} Q^{0}-\frac{t^{2}}{2} Q^{2}+t Q^{4}-Q^{6} .
$$

The comparison of (3.1) and (3.3) gives the relation between the low energy charges and the topological invariants of $\eta$. We derive next explicit formulae for the cases when $\eta$ describes either D6-branes wrapped on $X$ or D4-branes wrapped on submanifolds of $X$. More general situations (for example if $\eta$ is not representable by a sheaf on $X$ ) can be treated similarly.

2 Note that our conventions are such that D0-brane configurations have $\int_{X} \mathrm{ch}_{3}<0$. The associated $\mathrm{K}$ theory class is $-\left[\mathcal{O}_{P}\right]$ rather than $\left[\mathcal{O}_{P}\right]$, where $\mathcal{O}_{P}$ is a skyscraper sheaf of length one supported at the point $P$. Similarly, a D2-brane wrapping a curve $C \subset X$ is represented by the $\mathrm{K}$ theory class $-\left[\mathcal{O}_{C}\right]$ rather than $\left[\mathcal{O}_{C}\right]$. 


\subsection{The D6-Brane}

We now consider D-brane systems with nonzero D6-charge which can be represented by holomorphic vector bundles on $X$. In fact, it will turn out that this condition is too restrictive and we will actually have to enlarge the class of geometrical objects to coherent sheaves on $X$. Note also that the corresponding D-brane configuration is supersymmetric only if $V$ is a stable sheaf [11]. Expanding (3.2), we obtain

$$
Q=\left(r, c_{1}(V), \operatorname{ch}_{2}(V)+\frac{r}{24} c_{2}(X), \operatorname{ch}_{3}(V)+\frac{1}{24} c_{1}(V) c_{2}(X)\right)
$$

The associated central charge formula is therefore

$$
Z(Q)=\frac{r}{6} t^{3}-\frac{1}{2} \operatorname{ch}_{1}(V) t^{2}+\left(\operatorname{ch}_{2}(V)+\frac{r}{24} c_{2}(X)\right) t-\left(\operatorname{ch}_{3}(V)+\frac{1}{24} c_{1}(V) c_{2}(X)\right)
$$

A direct comparison of $(3.1)$ and $(3.5)$ yields

$$
\begin{aligned}
& r=n_{6} \\
& \operatorname{ch}_{1}(V)=n_{4}^{1} E+n_{4}^{2} S \\
& \operatorname{ch}_{2}(V)=\left(\frac{3}{2} n_{4}^{2}+n_{2}^{1}\right) h+\left(\frac{3}{2} n_{4}^{1}+n_{2}^{2}\right) l \\
& \operatorname{ch}_{3}(V)=-n_{0}+\frac{1}{2} n_{4}^{1}-3 n_{4}^{2} .
\end{aligned}
$$

Therefore

$$
\begin{aligned}
r=n_{6} & \\
c_{1}(V)= & n_{4}^{1} E+n_{4}^{2} S \\
c_{2}(V)= & {\left[\frac{1}{2}\left(n_{4}^{2}\right)^{2}-\frac{3}{2} n_{4}^{2}-n_{2}^{1}\right] h+} \\
& {\left[-\frac{3}{2}\left(n_{4}^{1}\right)^{2}+n_{4}^{1} n_{4}^{2}-\frac{3}{2} n_{4}^{1}-n_{2}^{2}\right] l } \\
c_{3}(V)= & \frac{1}{2}\left(3\left(n_{4}^{1}\right)^{3}-3\left(n_{4}^{1}\right)^{2} n_{4}^{2}+n_{4}^{1}\left(n_{4}^{2}\right)^{2}\right)+\frac{9}{2}\left(n_{4}^{1}\right)^{2}-3 n_{4}^{1} n_{4}^{2}- \\
& n_{4}^{1} n_{2}^{1}-\left(n_{4}^{2}-3 n_{4}^{1}\right) n_{2}^{2}-6 n_{4}^{2}+n_{4}^{1}-2 n_{0} .
\end{aligned}
$$

3 We will not give the explicit definition of stability here. The Donaldson-Uhlenbeck-Yau theorem shows that stability is essentially equivalent with self-duality of the gauge field configuration, which is more familiar to physicists. 
These formulae relate the topological invariants of the sheaf $V$ to the effective charges $\mathbf{n}$, as promised. In the next sections, they will play an important role in identifying the geometric properties of the Gepner model boundary states.

Before moving on to more general D-brane configurations, note that we can use the above explicit formulae to convert the monodromy transformations (2.11) into automorphisms of $K(X)$, as promised before. More precisely, we claim that the two monodromy transformations correspond to the following automorphisms

$$
[V] \rightarrow\left[V \otimes \mathcal{O}_{X}(H)\right], \quad[V] \rightarrow\left[V \otimes \mathcal{O}_{X}(S)\right]
$$

where $V$ is a coherent sheaf on $X$. To show this, note that tensoring by a line bundle $\mathcal{O}_{X}(D)$ changes the topological invariants of $V$ as follows

$$
\begin{aligned}
& r\left(V^{\prime}\right)=r(V) \\
& \operatorname{ch}_{1}\left(V^{\prime}\right)=\operatorname{ch}_{1}(V)+r D \\
& \operatorname{ch}_{2}\left(V^{\prime}\right)=\operatorname{ch}_{2}(V)+\operatorname{ch}_{1}(V) D+\frac{r}{2} D^{2} \\
& \operatorname{ch}_{3}\left(V^{\prime}\right)=\operatorname{ch}_{3}(V)+\operatorname{ch}_{2}(V) D+\frac{1}{2} \operatorname{ch}_{1}(V) D^{2}+\frac{r}{6} D^{3}
\end{aligned}
$$

where $V^{\prime} \simeq V \otimes \mathcal{O}_{X}(D)$. Using (3.6), a direct computation shows that the linear transformations acting on $\mathbf{n}$ corresponding to $D=H, S$ are precisely given by

$$
M(H)=S_{1}^{-1}, \quad M(S)=S_{2}^{-1},
$$

where $S_{1}, S_{2}$ are the monodromy matrices in (2.11). This proves the claim.

\subsection{D4-Brane}

A different class of D-brane states can be obtained by wrapping lower even dimensional branes on submanifolds of $i: D \hookrightarrow X$. In order to obtain supersymmetric configurations, $D$ has to be a holomorphic cycle [25,8]. Multiple brane configurations are described as above by a coherent sheaf $V$ on $D$, which is required to be stable. The associated K theory class in $K(X)$ is simply defined by the torsion coherent sheaf $i_{\star} V$ which is the extension of $V$ by zero to $X$. Then, the Mukai vector can be computed by a simple application of the Grothendieck-Riemann-Roch formula for the embedding $i: D \hookrightarrow X$

$$
i_{*}(\operatorname{ch}(V) \operatorname{Td}(D))=\operatorname{ch}\left(i_{*} V\right) \operatorname{Td}(X) .
$$


Note that this formula relates the topological invariants of the sheaf $V$ on $D$ to the topological invariants of the torsion sheaf $i_{\star} V$ on $X$. A direct expansion yields

$$
\begin{aligned}
& \operatorname{ch}_{1}\left(i_{*} V\right)=r D \\
& \operatorname{ch}_{2}\left(i_{*} V\right)=i_{*} c_{1}(V)+\frac{r}{2} i_{*} c_{1}(D) \\
& \operatorname{ch}_{3}\left(i_{*} V\right)=i_{*}\left(\operatorname{ch}_{2}(V)+\frac{1}{2} c_{1}(V) c_{1}(D)+\frac{r}{12}\left(c_{1}(D)^{2}+c_{2}(D)\right)\right)-\frac{r}{12} D c_{2}(X) .
\end{aligned}
$$

It follows that the Mukai charge vector is

$$
\begin{aligned}
Q=\left(0, r D, i_{*} c_{1}(V)+\frac{r}{2} i_{*} c_{1}(D), \operatorname{ch}_{2}(V)+\frac{1}{2} c_{1}(V) c_{1}(D)+\right. \\
\left.\frac{r}{12}\left(c_{1}(D)^{2}+c_{2}(D)\right)-\frac{r}{24} D c_{2}(X)\right) .
\end{aligned}
$$

In the last entry of the above formula, the top Chern classes are evaluated on the corresponding fundamental cycles, resulting in numerical invariants. Using an adjunction formula

$$
D c_{2}(X)=c_{2}(D)-D^{3}=c_{2}(D)-c_{1}(D)^{2},
$$

the charge vector can be rewritten

$$
Q=\left(0, r D, i_{*} c_{1}(V)+\frac{r}{2} i_{*} c_{1}(D), \operatorname{ch}_{2}(V)+\frac{1}{2} c_{1}(V) c_{1}(D)+\frac{r}{8} c_{1}(D)^{2}+\frac{r}{24} c_{2}(D)\right) .
$$

Therefore, in the large volume limit, the central charge associated to the charge vector (3.13) reads

$$
\begin{aligned}
Z(Q)= & -\frac{r}{2} t^{2} D+\left(i_{*} c_{1}(V)+\frac{r}{2} i_{*} c_{1}(D)\right) t-\operatorname{ch}_{2}(V)- \\
& \frac{1}{2} c_{1}(V) c_{1}(D)-\frac{r}{8} c_{1}(D)^{2}-\frac{r}{24} c_{2}(D) .
\end{aligned}
$$

Note that $i_{*} c_{1}(V), i_{*} c_{1}(D)$ can be thought as curve classes on $X$ by Poincaré duality, therefore the intersection number in the second term is well defined. With an eye on applications, we derive explicit formulae for multiple D4-branes with lower induced charges wrapping the cycles $E$ and $S$ on the elliptic fibration.

Branes wrapped on $E$ correspond to BPS states with charge vectors of the form $\mathbf{n}=\left(0, n_{4}^{1}, 0, n_{0}, 0, n_{2}^{2}\right)$ with central charge

$$
Z(\mathbf{n})=n_{4}^{1} \mathcal{F}^{1}+n_{2}^{2} t_{2}+n_{0}
$$

4 Note that the allowed lower D2-brane charges correspond to cycles that can be embedded in E. 
The topological invariants of the sheaf $V$ can be obtained from equations (3.16) and (3.17)

$$
\begin{aligned}
& r=n_{4}^{1} \\
& \operatorname{ch}_{1}(V)=n_{2}^{2} l \\
& \operatorname{ch}_{2}(V)=-n_{4}^{1}-\frac{3}{2} n_{2}^{2}-n_{0} .
\end{aligned}
$$

Therefore

$$
\begin{aligned}
& r=n_{4}^{1} \\
& c_{1}(V)=n_{2}^{2} l \\
& c_{2}(V)=\frac{1}{2} n_{2}^{2}\left(n_{2}^{2}+3\right)+n_{4}^{1}+n_{0} .
\end{aligned}
$$

Similarly, branes wrapping $S$ correspond to BPS charge vectors of the form $\mathbf{n}=$ $\left(0,0, n_{4}^{2}, n_{0}, n_{2}^{1}, n_{2}^{2}\right)$ with central charge

$$
Z(\mathbf{n})=n_{4}^{2} \mathcal{F}^{2}+n_{2}^{1} t_{1}+n_{2}^{2} t_{2}+n_{0} .
$$

Using again (3.16) and (3.20), we deduce

$$
\begin{aligned}
& r=n_{4}^{2} \\
& \operatorname{ch}_{1}(V)=\left(2 n_{4}^{2}+n_{2}^{1}\right) h+n_{2}^{2} l \\
& \operatorname{ch}_{2}(V)=-3 n_{4}^{2}+\frac{1}{2} n_{2}^{2}-n_{0} .
\end{aligned}
$$

Therefore

$$
\begin{aligned}
& r=n_{4}^{2} \\
& c_{1}(V)=\left(2 n_{4}^{2}+n_{2}^{1}\right) h+n_{2}^{2} l \\
& c_{2}(V)=-\frac{1}{2} n_{2}^{2}\left(3 n_{2}^{2}+1\right)+2 n_{2}^{2} n_{4}^{2}+n_{2}^{1} n_{2}^{2}+3 n_{4}^{2}+n_{0} .
\end{aligned}
$$

More general configurations involving various configurations of D4-branes and D2-branes on holomorphic cycles in $X$ can be treated similarly.

\section{Boundary States in the Gepner Model}

As explained before, the Gepner point is a special point in the moduli space where the superconformal field theory is exactly solvable [26,27,28]. This feature allows us to obtain valuable information on the spectrum of BPS states in a deep stringy regime of the theory. In the present section, we explain the construction of B type boundary state in the 
$(k=16)^{3}(k=1)$ Gepner model which is continuously connected to the elliptic Calabi-Yau compactification considered in the previous sections. The construction is closely related to the one applied to rational conformal field theories in [29] and it has been considered in detail in [4, 5, 6]. Our approach is focused on the conformal field theory computation of the symplectic intersection form on the BPS charge lattice [30]. As in [14], this is a crucial ingredient in the geometric interpretation of Gepner model boundary states.

The B type boundary states at the Gepner point $(k=16)^{3}(k=1)$ are labeled as in [14] by $\left|L_{1}, L_{2}, L_{3}, L_{4} ; M ; S\right\rangle$ where $0 \leq L_{1}, L_{2}, L_{3} \leq 8, L_{4}=0, M \in \mathbf{Z}_{36}$

$$
M=\sum_{i=1}^{4} \frac{K^{\prime} M_{i}}{k_{i}+2}
$$

with $K^{\prime}=$ l.c.m. $\left\{k_{i}+2\right\}$ and $S \in 2 \mathbf{Z}_{2}$. For fixed $L=\left(L_{i}\right)$, the states with different $(M, S)$ form an orbit under the $\mathbf{Z}_{18}$ discrete symmetry group and the two values of $S$ correspond to a brane and the corresponding antibrane.

\subsection{The Intersection Form}

A first step in deriving a geometric interpretation of the Gepner model boundary states is to calculate their intersection numbers using exact conformal field theory techniques. As explained in [31,30,14, these numbers can be obtained by computing $\operatorname{tr}(-1)^{F}$ in the internal part of the open string $\mathrm{R}$ sector. Since this quantity is an index, it is unchanged under marginal deformations of the SCFT on the moduli space. Therefore it can be reliably compared with the large radius limit intersection matrix given by (2.9) in a basis adapted to the $\mathbf{Z}_{18}$ symmetry of the Gepner point. An explicit calculation gives

$$
I_{B}=\frac{1}{C}(-1)^{\frac{S-\tilde{S}}{2}} \sum_{m_{j}^{\prime}} \delta_{\frac{M-\tilde{M}}{2}+\sum \frac{K^{\prime}}{2 k_{j}+4}\left(m_{j}^{\prime}+1\right)}^{\left(K^{\prime}\right)} \prod_{j=1}^{r} N_{L_{j}, \tilde{L}_{j}}^{m_{j}^{\prime}-1},
$$

where $N_{L, \tilde{L}}^{l}$ are the $S U(2)_{k}$ fusion rule coefficients. This formula is very cumbersome, but it can be rewritten in a much simpler form. To this end one can note that the states within one $\mathbf{Z}_{18}$ orbit $\left(L_{j}\right.$ are fixed) can be labeled by a 36 dimensional row vector $q_{B}$ with all entries equal to zero, except for the $M$ th entry, which equals one.

In this notation the intersection matrices for boundary states in fixed orbits of the $\mathbf{Z}_{18}$ symmetry can be expressed in terms of shift matrices $g$. Each factor $N_{L_{j}, \tilde{L}_{j}}^{m_{j}^{\prime}-1}$ in (4.2) can be replaced in matrix notation by a factor 5

$$
n_{L, \tilde{L}}=n_{\tilde{L}, L}=g^{\frac{|L-\tilde{L}|}{2}}+g^{\frac{|L-\tilde{L}|}{2}+1}+\cdots+g^{\frac{L+\tilde{L}}{2}}-g^{-1-\frac{|L-\tilde{L}|}{2}}-\cdots-g^{-1-\frac{L+\tilde{L}}{2}} .
$$

5 The matrix $g^{\frac{1}{2}}$ is understood as the basic 36 -dimensional shift matrix. 
The delta function constraint is a shifted $U(1)$ projection, showing that the intersection matrix is $\mathbf{Z}_{18}$ invariant.

As specified before, the BPS charge vectors associated to the Gepner model boundary states can be found by comparing the intersection matrices (4.2) and (2.10). It turns out that it is more convenient to work in a different basis for boundary states, related to the present one by the linear transformation $\frac{1}{\sqrt{2}}\left(1-g^{9}\right)$. In the new basis, the matrix (4.2) reads

$$
I_{B}=\left(1-g^{17}\right)^{3}\left(1-g^{12}\right)\left(1-g^{9}\right) .
$$

This is antisymmetric and easy combinatorics shows that it's rank is 6. From (2.10) and (4.4) it can be seen that the two matrices $I_{G}$ and $I_{B}$ are related by

$$
I_{B}=(1-g) I_{G}(1-g)^{t}
$$

This connects the series of boundary states with $\left(L_{j}\right)=(0, \cdots, 0)$ to the basis of periods $\omega$ at the Gepner point.

To find the charges of boundary states with $L_{j} \neq 0$ one has to find a linear transformation $t_{L}$ which generates the different factors $n_{L, \tilde{L}}$ from $n_{0,0}=\left(1-g^{-1}\right)$. The change of basis is expressible in terms of $g$

$$
t_{L}=t_{L}^{t}=\sum_{l=-\frac{L}{2}}^{\frac{L}{2}} g^{l}
$$

The first step in verifying this is to relate $n_{L, 0}$ to $n_{0,0}$

$$
t_{L} n_{0,0}=\sum_{l=-\frac{L}{2}}^{\frac{L}{2}} g^{l}\left(1-g^{-1}\right)=\sum_{l=-\frac{L}{2}}^{\frac{L}{2}} g^{l}-\sum_{l=-\frac{L}{2}-1}^{\frac{L}{2}-1} g^{l}=g^{\frac{L}{2}}-g^{-\frac{L}{2}-1}=n_{L, 0} .
$$

Multiplying this with $t_{\tilde{L}}$ one gets

$$
t_{L} n_{0,0} t_{\tilde{L}}^{t}=\sum_{l=-\frac{L}{2}}^{\frac{L}{2}} g^{l}\left(g^{\frac{\tilde{L}}{2}}-g^{-\frac{\tilde{L}}{2}-1}\right)=\sum_{l=\frac{|L-\tilde{L}|}{2}}^{\frac{L+\tilde{L}}{2}}\left(g^{l}-g^{-l-1}\right)=n_{L, \tilde{L}} .
$$

The charge of the boundary state $q_{B}$ in the Gepner basis is then given by $q_{G}=$ $q_{B} t_{L_{1}} t_{L_{2}} t_{L_{3}}(1-g)$. This row vector has only entries in the even columns, which means that all the odd columns can be omitted, leaving a 18 dimensional vector. There are only 6 independent charges and the relations (2.7) can be used to reduce $q_{G}$ to it's first 6 entries. The large radius charge $q_{L}$ is then easily calculated from the reduced $q_{B}$ by $q_{L}=q_{G} m$. 


\subsection{Marginal Operators}

For comparison with geometric results it will be interesting to compute the number of boundary marginal operators. As explained in [14 they can be expressed in terms of the matrices $\tilde{n}_{L, \tilde{L}}=\left|n_{L, \tilde{L}}\right|$. The number of boundary marginal operators for only one boundary state $\left|L_{j}, M, S\right\rangle$ is given by the diagonal part of

$$
\frac{1}{2} \tilde{n}_{L_{1}, L_{1}} \tilde{n}_{L_{2}, L_{2}} \tilde{n}_{L_{3}, L_{3}}\left(1+g^{12}\right)\left(1+g^{9}\right)-\# \text { vac. }
$$

The number of vacua is normally 1 and for each $L_{j}=8$ it is multiplied by 2 . The following table shows the number of marginal operators for some important boundary states

$\begin{array}{ccc}\left(L_{1}, L_{2}, L_{3}\right) & \# \text { (marg) } & \#(\text { vac }) \\ (0,0,0) & 0 & 1 \\ (1,0,0) & 2 & 1 \\ (2,0,0) & 3 & 1 \\ (3,0,0) & 3 & 1 \\ (4,0,0) & 3 & 1 \\ (5,0,0) & 3 & 1 \\ (6,0,0) & 4 & 1 \\ (7,0,0) & 6 & 1 \\ (8,0,0) & 6 & 2\end{array}$

These boundary operators are massless, but they might have a superpotential, with flat directions corresponding to the truly marginal operators.

If the number of vacua in the open string sector is different from zero, e.g. two, one might think of the boundary state as two different D-branes. This would fit with the picture of a Coulomb branch in the world volume theory in which the gauge group is $U(1)^{2}$. The reason that these boundary states appear in the formalism of the Gepner model as a single boundary state could be related to the higher symmetry algebra that these boundary states respect.

\section{Boundary States and Vector Bundles}

In this final section, we collect the results obtained so far and establish an explicit connection between Gepner model D-branes and supersymmetric brane configurations on the elliptically fibered Calabi-Yau variety. To summarize, this process essentially involves two stages. First, we interpret the boundary states at the Gepner point as generic BPS states on the moduli space. The next step involves a translation of the BPS charge vectors $\mathbf{n}$ 
into microscopic D-brane charges using the Chern-Simons couplings as explained in section three. Note that this procedure involves analytic continuation between two distinct regions of the moduli space, therefore the spectrum of BPS states may be affected by jumping and marginal stability phenomena. We will not attempt to give a comprehensive study of these issues here, but the geometric picture will eventually provide significant information on the stability of the Gepner model D-branes in the large radius limit. Moreover, we will also compare the number of moduli of a given D-brane configuration in the two regimes, finding a remarkable agreement. This suggests that the marginal deformations found in section 4.2 are in fact truly marginal and the corresponding flat directions are not lifted by superpotential couplings. Note that an exhaustive treatment of all Gepner boundary states is not possible due to their large number (1485, according to some combinatorial arguments). Therefore we will restrict in the following to a subset of states which admit a simple geometric interpretation.

\section{1. $L=(0000)$}

The simplest series is $L=(0000)$. The 18 states forming a $\mathbf{Z}_{18}$ orbit are grouped in D-brane/anti-D-brane pairs, therefore there are only 9 relevant charge vectors, which are listed in the following table

$\begin{array}{ccccccc}\text { No } & n_{6} & n_{4}^{1} & n_{4}^{2} & n_{0} & n_{2}^{1} & n_{2}^{2} \\ \mathbf{n}_{1} & 1 & 0 & 0 & 0 & 0 & 0 \\ \mathbf{n}_{2} & 2 & 0 & -1 & 3 & 1 & 0 \\ \mathbf{n}_{3} & 1 & -1 & -1 & 3 & 2 & 1 \\ \mathbf{n}_{4} & 1 & -1 & 0 & 1 & 0 & 0 \\ \mathbf{n}_{5} & 1 & 0 & -1 & 3 & 2 & 0 \\ \mathbf{n}_{6} & 0 & 1 & 0 & -1 & 0 & 0 \\ \mathbf{n}_{7} & 0 & 2 & 0 & 0 & 0 & -1 \\ \mathbf{n}_{8} & 0 & 1 & 0 & 0 & 0 & -1 \\ \mathbf{n}_{9} & 2 & -2 & -1 & 3 & 1 & 1 .\end{array}$

The corresponding topological invariants can be computed by a direct application of the formulae in sections 3.1 and 3.2. We find that the states $\mathbf{n}_{1}, \mathbf{n}_{3}, \mathbf{n}_{4}, \mathbf{n}_{5}$ correspond to the complex holomorphic line bundles $\mathcal{O}_{X}, \mathcal{O}_{X}(-E-S), \mathcal{O}_{X}(-E), \mathcal{O}_{X}(-S)$ respectively. These are clearly stable and describe supersymmetric single D6-brane configurations with induced anti-D4 charges. They are also rigid, since $h^{0,1}(X)=0$, therefore the number of moduli is zero, in agreement with the results in 3.5. Similarly, the states $\mathbf{n}_{6}$ and $\mathbf{n}_{8}$ correspond to the holomorphic line bundles $\mathcal{O}_{E}$ and $\mathcal{O}_{E}(-l)$ on the section $E \simeq \mathbf{P}^{2}$ of the 
elliptic fibration. These are again stable and rigid and correspond to single D4-branes on E.

The remaining states, $\mathbf{n}_{2,7,9}$ are more interesting since they correspond to multiple branes. The first charge is associated to a vector bundle $V$ on $X$ with topological invariants

$$
r(V)=2, \quad c_{1}(V)=-S, \quad c_{2}(V)=h, \quad c_{3}(V)=0 .
$$

A holomorphic vector bundle with these characteristics can be easily constructed as a pull back of a rank two vector bundle $W$ on the base $\mathbf{P}^{2}, V=\pi^{*} W$. For $W$, we find

$$
r(W)=2, \quad c_{1}(W)=-l, \quad c_{2}(W)=1
$$

and it turns out that this is an exceptional bundle 6 on the projective plane $\mathbf{P}^{2}$ 32, 33. In particular, $W$ is stable and rigid. We can prove that $V$ is also rigid as follows. The infinitesimal deformations of $V$ on $X$ are parameterized by a the cohomology group

$$
H^{1}(X, \operatorname{End}(V))=H^{1}\left(X, V \otimes V^{*}\right)=H^{1}\left(X, \pi^{*}\left(W \otimes W^{*}\right)\right) .
$$

This can be evaluated using the Leray spectral sequence for $\pi: X \rightarrow B$

$$
H^{1}\left(X, \pi^{*}\left(W \otimes W^{*}\right)\right) \simeq H^{0}\left(B, W \otimes W^{*} \otimes K_{B}\right) \oplus H^{1}\left(B, W \otimes W^{*}\right) .
$$

Using Kodaira-Serre duality, we have

$$
\begin{aligned}
& H^{0}\left(B, W \otimes W^{*} \otimes K_{B}\right) \simeq H^{2}(B, \operatorname{End}(W))^{*}=0 \\
& H^{1}\left(B, W \otimes W^{*}\right)=H^{1}(B, \operatorname{End}(W))=0,
\end{aligned}
$$

since $W$ is exceptional. Therefore $V$ is indeed rigid and it can be proved similarly that $V$ is also simple i.e. it has no nontrivial automorphisms $\operatorname{Hom}(V, V) \simeq \mathbf{C}$. The stability of $V$ is harder to analyze and we have not been able to obtain a definite result.

Next, it can be checked that the state $\mathbf{n}_{7}$ represents a D4-brane with multiplicity two wrapped on the section $E$. The associated vector bundle turns out to be in fact isomorphic to the exceptional bundle $W$ considered in the previous paragraph. This is rigid and stable, therefore we obtain a supersymmetric configuration with no moduli. Finally, the ninth state corresponds to a bundle $V$ with topological invariants

$$
r(V)=2, \quad c_{1}(V)=-2 E-S, \quad c_{2}(V)=h-2 l, \quad c_{3}=0 .
$$

6 Exceptional bundles on a surface are in general characterized by $H^{1}(\operatorname{End}(W)) \simeq 0$ and $H^{2}(\operatorname{End}(W)) \simeq 0$. On the projective plane, one can prove that this implies stability. 
A bundle with these topological invariants can be easily constructed as $V \simeq \pi^{\star} W \otimes$ $\mathcal{O}_{X}(-E)$. It's properties are similar to those of $\pi^{\star} W$ i.e. $V$ is rigid and simple.

To summarize the results, we have found that all states, except possibly $\mathbf{n}_{2}, \mathbf{n}_{7}$ and $\mathbf{n}_{9}$, are stable and supersymmetric in the large radius limit. Moreover, the number of geometric moduli agrees with the number of marginal deformations of the boundary states. This provides supporting evidence for the decoupling of the bulk Kähler moduli argued in [14. The states $\mathbf{n}_{2}, \mathbf{n}_{7}$ and $\mathbf{n}_{9}$ are more intriguing since we have not been able to settle the issue of stability in the large radius limit. On the other hand, since the associated bundles are simple, these D-brane configurations should correspond to one particle states in the four dimensional effective theory. If the bundles turn out to be unstable, it would be interesting to understand if these are stable non-BPS states in the large radius limit.

Next, we consider another group of Gepner model boundary states which lead to more interesting physical configurations.

\section{2. $L=(2000)$}

The charge vectors of the states in this series are

$\begin{array}{ccccccc}\mathbf{n} & n_{6} & n_{4}^{1} & n_{4}^{2} & n_{0} & n_{2}^{1} & n_{2}^{2} \\ \mathbf{n}_{1} & 0 & 0 & 0 & 1 & 0 & 0 \\ \mathbf{n}_{2} & 0 & 0 & 0 & 0 & -1 & 0 \\ \mathbf{n}_{3} & 0 & 0 & 0 & 1 & -1 & 0 \\ \mathbf{n}_{4} & 1 & 0 & 0 & 1 & 0 & 0 \\ \mathbf{n}_{5} & 1 & -1 & 0 & 1 & -1 & 0 \\ \mathbf{n}_{6} & 1 & 0 & -1 & 2 & 1 & 0 \\ \mathbf{n}_{7} & 1 & -1 & -1 & 2 & 2 & 1 \\ \mathbf{n}_{8} & 0 & 1 & 0 & 0 & 1 & 0 \\ \mathbf{n}_{9} & 0 & 1 & 0 & 0 & -1 & -1 .\end{array}$

The first charge vector in this series is particularly interesting since it corresponds to a D0-brane on $X$. Formally, this is described by a $\mathrm{K}$ theory class $\eta \in K(X)$ with topological invariants

$$
r(\eta)=0, \quad \operatorname{ch}_{1}(\eta)=0, \quad \operatorname{ch}_{2}(\eta)=0, \quad \operatorname{ch}_{3}(\eta)=-1 .
$$

This identifies $\eta$ as $-\left[\mathcal{O}_{P}\right]$ where $\mathcal{O}_{P}$ is a skyscraper sheaf of length one supported at the point $P \in X$. The next two states $\mathbf{n}_{2,3}$ also have a simple physical interpretation. They correspond to a D2-brane wrapped on the elliptic fiber and respectively to a D2-brane wrapped on the elliptic fiber with a magnetic flux inducing one unit of D0-charge. The $\mathrm{K}$ theory classes can be easily constructed. Pick $i: Y \hookrightarrow X$ to be an arbitrary smooth 
elliptic fiber in the class $h$ and pick $\mathcal{L}_{Y}$ to be a holomorphic line bundle on $Y$. We must have $\operatorname{deg}\left(\mathcal{L}_{Y}\right)=0$ for $\mathbf{n}_{2}$ and $\operatorname{deg}\left(\mathcal{L}_{Y}\right)=1$ for $\mathbf{n}_{3}$. Then the required $\mathrm{K}$ theory class is $\eta=-\left[i_{\star} \mathcal{L}_{Y}\right]$. All these configurations are supersymmetric and we can also determine the moduli space. For a D0-brane, the moduli space is simply isomorphic to $X$. For the D2-branes, the moduli are parameterized by a point in the base $\mathbf{P}^{2}$ representing the projection of the elliptic fiber $Y$ and the choice of a flat line bundle on $Y$ (specifying the Wilson lines). Therefore the global moduli space for D2-branes is isomorphic to the relative Jacobian variety of $X$, which is in turn isomorphic to $X$ itself since the elliptic fibration has only nodal and cuspidal fibers. Moreover, the number of moduli agrees with the number of marginal deformations computed at the Gepner point, supporting again the decoupling of Kähler moduli argued in [14].

Next, the states $\mathbf{n}_{4} \ldots \mathbf{n}_{7}$ have one unit of D6-charge. $\mathbf{n}_{4}$ is particularly interesting since the topological invariants

$$
r(\eta)=1, \quad \operatorname{ch}_{1}(\eta)=0, \quad \operatorname{ch}_{2}(\eta)=0, \quad \operatorname{ch}_{3}(\eta)=-1
$$

identify the ideal sheaf of a single point $P$ on $X$. In the present conventions, this corresponds to a D6-D0 system which is very interesting. In flat space it is known that D6-D0 systems are repulsive and they break supersymmetry completely. On a curved manifold, the open string dynamics is harder to analyze, but in the large radius limit, the D6-D0 potential should approach continuously the flat space result. Therefore, we expect this configuration to be nonsupersymmetric and repulsive at sufficiently large radius. In particular, the repulsive interaction prevents the occurrence of a bound state. This gives a clear example supersymmetric Gepner model state which decays in a nonsupersymmetric combination of D-branes in the large radius limit. Such phenomena have been predicted in [14]. This picture is especially interesting when interpreted from the mirror $\hat{X}$ point of view. As also mentioned in the introduction, we obtain an example of a phase transition of special lagrangian cycles as we move on the complex structure moduli space of $\hat{X}$. Regarding mirror symmetry as T-duality as in [16], $X$ and $\hat{X}$ admit special lagrangian fibrations with dual $T^{3}, \hat{T}^{3}$ fibers. Then the D6 and D0-branes on $X$ are mapped to D3-branes wrapping the base $B$ of the fibration and respectively the $\hat{T}^{3}$ fiber. The previous argument shows that the homology class $B+\hat{T}^{3}$ should not support a special lagrangian cycle in a neighborhood of the large complex structure limit of $\hat{X}$. However, it should support such a cycle in a region of the moduli space of $\hat{X}$ which maps to a neighborhood of the 
Gepner point of $X$ under the mirror map. This predicts transitions of the type discussed in [17] in a concrete compact model.

The remaining states $\mathbf{n}_{5,6,7}$ correspond to various D6, D4 and D2 combinations whose existence as bound states is an open problem. The topological charges can be easily computed as above, but we will not pursue this here.

Finally, the last two states $\mathbf{n}_{8,9}$ represent configurations with a D4-brane wrapped on the section $E$ of the elliptic fibration and a D2-brane wrapped on an elliptic fiber $Y$. For $\mathbf{n}_{9}$, the D4-brane carries a magnetic flux on a hyperplane in $\mathbf{P}^{2}$ which induces a lower D2brane charge. Here, the dynamics is very similar to that of the D6-D0 system (in fact they can be related by a T-duality on the elliptic fiber). As $X$ approaches the large radius limit, the brane systems approach configurations of transverse D4-D2 branes in flat space. These are nonsupersymmetric and repulsive, therefore they cannot form bound states. Hence, we find more examples of Gepner model states which decay into non-BPS configurations in the large volume limit. They should be interpreted in terms of phase transitions of special-lagrangian cycles on $\hat{X}$, as before.

The other series of Gepner model boundary states can be analyzed similarly, resulting in various brane configurations. We will not pursue this systematically here, but we would like to emphasize two other states which have not appeared in the previous cases. Namely, in the series $L=(2100)$ (which is identical to $L=(7000)$ ), one finds the charge vectors $\mathbf{n}_{1}=$ $(0,0,1,-1,-3,-1)$ and $\mathbf{n}_{2}=(0,0,1,-1,-1,0)$ which correspond to D4-branes wrapping a vertical holomorphic four-cycle $i: D \hookrightarrow X$ in the class $S$. Note that the surfaces in this class are generically smooth elliptic fibrations over a rational curve in the class $l$. The associated $\mathrm{K}$ theory classes are determined by the torsion sheaves $i_{\star}\left(J_{P+Q} \otimes \mathcal{O}_{D}(-h-l)\right)$ and $i_{\star}\left(J_{P+Q} \otimes \mathcal{O}_{D}(h)\right)$, where $J_{P+Q}$ is the ideal sheaf of two (possible coincident) points on $D$. Therefore, in both cases, we obtain a single D4-brane wrapped on $D$ with two units of D0-charge which correspond to D0-branes located at the points $P, Q$. The D4-branes also carry magnetic flux inducing lower D2-brane charges on curves in the class $-h-l$ and respectively $h$. The existence of bound states with these charges is an open problem, but the large radius limit analysis suggests that they might exist since the D4-D0-systems are supersymmetric in flat space. In fact, the bound states would have to be marginal, which makes the existence problem very subtle.

\section{Acknowledgments}

We are very grateful to Michael Douglas for suggesting the problem and for collabo- 
ration at an early stage of this work. We would also like to thank Ilka Brunner, Jaume Gomis, Paul Horja, Albrecht Klemm, Greg Moore and Ronen Plesser for valuable discussions. The work of D.-E. D. has been supported by DOE grant DE-FG02-90ER40542 and the work of C. R. has been supported by DOE grant DE-FG02-96ER40559. 


\section{References}

[1] J. Dai, R. Leigh, and J. Polchinski, "New Connections Between String Theories", Mod. Phys. Lett. A4 (1989) 2073;

R. Leigh, "Dirac-Born-Infeld Action from Dirichlet Sigma Model", Mod. Phys. Lett. A4 (1989) 2767;

P. Horava, "Strings on World-Sheet Orbifolds," Nucl. Phys. B327 (1989) 461;

P. Horava, "Background Duality of Open String Models", Phys. Lett. B231 (1989) 251 ;

M.B. Green, "Space-time Duality and Dirichlet String Theory", Phys. Lett. B266 325 (1991), "Pointlike States for Type IIB Superstrings", Phys. Lett. B329 (1994) 435, hep-th/9403040; "A Gas of D Instantons", Phys. Lett. B354 (1995) 271, hepth/9504108;

J. Polchinski, "Combinatorics of Boundaries in String Theory", Phys. Rev. D50 (1994) 6041.

[2] R. Minasian and G. Moore, "K-theory and Ramond-Ramond charge", JHEP 11 (1997) 002, hep-th/9710230.

[3] E. Witten, "D-Branes and K Theory", JHEP 12 (1998) 019, hep-th/9810188.

[4] A. Recknagel and V. Schomerus, "D-branes in Gepner models," Nucl. Phys. B531, 185 (1998) hep-th/9712186.

[5] M. Gutperle and Y. Satoh, "D-branes in Gepner models and supersymmetry", Nucl. Phys. B543 (1999) 73, hep-th/9808080.

[6] M. Gutperle and Y. Satoh, "D0-branes in Gepner models and N=2 black holes", Nucl. Phys. B555 (1999) 477, hep-th/9902120.

[7] M.R. Douglas and B. Fiol, "D-Branes and Discrete Torsion II", hep-th/9903031.

[8] H. Ooguri, Y. Oz, Z. Yin, "D-Branes on Calabi-Yau Spaces and Their Mirrors", Nucl. Phys. B477 (1996) 407, hep-th/9606112.

[9] M.R. Douglas, "Branes within Branes", contributed to "Cargese 1997, Strings, Branes and Dualities", 267-275, hep-th/9512077.

[10] M. Green, J.A. Harvey and G. Moore, "I-Brane Inflow and Anomalous Couplings on D-Branes", Class. Quant. Grav. 14 (1997) 47, hep-th/9605033.

[11] J.A. Harvey and G. Moore, "On The Algebras of BPS States", Commun. Math. Phys. 197 (1998) 489, hep-th/9609017.

[12] Y.-K E.Cheung and Z. Yin, "Anomalies, Branes and Currents", Nucl. Phys. B517 (1998) 69, hep-th/9710206.

[13] P. Candelas, A. Font, S. Katz and D.R. Morrison, "Mirror symmetry for two parameter models. 2," Nucl. Phys. B429, 626 (1994) hep-th/9403187.

[14] I. Brunner, M.R. Douglas, A. Lawrence and C. Römelsberger, "D-branes on the quintic," hep-th/9906200. 
[15] D.-E. Diaconescu and J. Gomis, "Fractional Branes and Boundary States in Orbifold Theories", hep-th/9906242.

[16] A. Strominger, S.-T. Yau, E. Zaslow, "Mirror Symmetry is T-Duality", Nucl. Phys. B479 (1996) 243, hep-th/9606040.

[17] D. Joyce, "On counting special Lagrangian homology 3-spheres", hep-th/9907013.

[18] S. Kachru and J. McGreevy, "Supersymmetric Three-cycles and (Super)symmetry Breaking", hep-th/9908135.

[19] B.R. Greene and M.R. Plesser, "Duality In Calabi-Yau Moduli Space," Nucl. Phys. B338 (1990) 15.

[20] M. Kontsevich, "Homological Algebra of Mirror Symmetry", alg-geom/9411018.

[21] A. Polishchuk and E. Zaslow, "Categorical Mirror Symmetry: The Elliptic Curve", alg-geom/9801119.

[22] C. Vafa, "Extending Mirror Conjecture to Calabi-Yau with Bundles", hep-th/9804131.

[23] C. Bartocci, U. Bruzzo and G. Sanguinetti, "Categorial mirror symmetry for K3 surfaces", math-ph/9811004.

[24] Paul Horja, dissertation thesis, Duke University.

[25] K. Becker, M. Becker and A. Strominger, "Fivebranes, Membranes and NonPerturbative String Theory", Nucl. Phys. B456 (1995) 130, hep-th/9507158.

[26] E. Witten, "Phases of $\mathrm{N}=2$ theories in two dimensions," Nucl. Phys. B403, 159 (1993) hep-th/9301042.

[27] W. Lerche, C. Vafa and N.P. Warner, "Chiral Rings In N=2 Superconformal Theories," Nucl. Phys. B324, 427 (1989).

[28] E. Witten, "On the Landau-Ginzburg description of $\mathrm{N}=2$ minimal models," Int. J. Mod. Phys. A9, 4783 (1994) hep-th/9304026.

[29] J.L. Cardy, "Boundary Conditions, Fusion Rules And The Verlinde Formula," Nucl. Phys. B324, 581 (1989).

[30] M.R. Douglas and B. Fiol, "D-branes and discrete torsion. II", hep-th/9903031.

[31] M. Berkooz and M. Douglas, "Five-branes in M(atrix) theory," Phys. Lett. B395, (1997) 196, hep-th/9610236.

[32] J.-M. Drézet and J. Le Poitier, "Fibrés Stable et fibrés Exceptionnels sur Le Plan Projectif", Ann. Scient. Ec Norm. Sup. 18 (1998) 105.

[33] J. Le Poitier, "Lectures on Vector Bundles", Cambridge University Press, 1997. 